\title{
REGENERAÇÃO NATURAL NA FLORESTA OMBRÓFILA MISTA SUBMETIDA A TRATAMENTOS DE CONTROLE DE TAQUARA
}

\section{Natural Regeneration in Araucaria Forest Submitted to Bamboo Control Treatments}

\author{
Ana Paula Dalla Corte \\ Doutoranda em Eng. Florestal, UFPR, Curitiba - PR. e-mail: anacorte@ufpr.br \\ Dirceu Lúcio Carneiro de Miranda \\ Mestrando em Eng. Florestal, UFPR, Curitiba - PR. e-mail: mirandaufpr@yahoo.com.br \\ Karla Simone Weber \\ Mestranda em Eng. Florestal. e-mail: karlasimone@gmail.com \\ Elenise Leocádia da Silveira Nunes \\ Mestranda em Eng. Florestal, UFPR, Curitiba - PR. e-mail: elenise.nunes@bol.com.br \\ Carlos Roberto Sanquetta \\ Eng. Florestal, Ph.D., Depto. de Ciências Florestais, UFPR, Curitiba - PR. e-mail: sanquetta@ufpr.br \\ Roberto da Conceição Ferreira \\ Doutorando em Eng. Florestal, UFPR, Curitiba - PR. e-mail: robertoferre@bol.com.br
}

\begin{abstract}
Resumo
Este trabalho teve por objetivo avaliar estatisticamente a regeneração natural em Floresta Ombrófila Mista das espécies arbóreas vassourão-branco (Piptocarpha angustifolia Dusén ex Malme), vassourãopreto (Vernonia discolor Spreng.), capororoca (Myrsine umbellata Mart.), erva-mate (Ilex paraguariensis St. Hil), pau-de-anta (Symplocos tenuifolia Brand.), entre outras, submetidas a cinco diferentes tratamentos de controle mecânico de taquaras, sendo analisadas as variáveis número de indivíduos e altura média dos indivíduos de cada espécie. Em 25 unidades experimentais de 20 x 20 metros, foi utilizado delineamento inteiramente casualizado, aplicando os seguintes tratamentos com 5 repetições: testemunha; corte total da taquara e abandono; corte total da taquara e retirada; corte total da taquara e abandono (duas vezes); corte total da taquara + corte subdossel $(C A P<31,5 \mathrm{~cm})$, sem retirada. As análises estatísticas empregadas foram o Teste de Bartlett, para verificar a homogeneidade das variâncias, a ANOVA para verificar variações significativas e o Teste de Tukey, para identificar entre quais grupos as diferenças ocorreram. Em relação ao número de indivíduos, o resultado da análise de variância demonstrou que Myrsine umbellata e Piptocarpha angustifolia apresentaram diferenças significativas entre as médias em relação aos tratamentos aplicados. Das análises estatísticas realizadas, a espécie Myrsine umbellata foi a única que apresentou homogeneidade em suas variâncias, para a variável altura das regenerações naturais. Para as demais espécies, a variabilidade foi alta, razão pela qual se efetuou apenas uma análise visual gráfica sobre tais dispersões.
\end{abstract}

Palavras-chave: Araucaria angustifolia; Taquara; Regeneração natural; Espécies florestais.

$\overline{\text { Esta publicação foi realizada com apoio do } \mathrm{MCT} / \mathrm{CNPq}}$. 


\begin{abstract}
The objective of this research was to evaluate statistically the natural regeneration of Piptocarpha angustifolia Dusén ex Malme, Vernonia discolor (Spreng.) Less., Myrsine umbellata Mart., Ilex paraguariensis St. Hil, (Symplocos tenuifolia Brand.), and others, submitted to 5 treatments of bamboo control, where it was analyzed the variables number of plants per plot and the average height of plants. In 25 sample plots with $20 \times 20$ meters, it was used completely randomized design $(C R D)$, applying the following treatments with 5 replications: control; total cut of bamboo and leaving it in the forest; total cut of bamboo and its removing; twice total cut of bamboo and its removing; total cut of bamboo, understory $(C B H<31.5 \mathrm{~cm})$ and no removing of them. It was used Bartlett test, ANOVA and Tukey test for the data analysis. About the number of plants, the ANOVA showed that there were significant differences among the treatments means for Piptocarpha angustifolia and Myrsine umbellata. As result of the statistical analyses, the Myrsine umbellata was the only one that presented homogeneity of variances, for the variable natural regeneration height. For the remaining species the height variability was high, reason why it was made only a graphical analysis of such dispersions.
\end{abstract}

Keywords: Araucaria angustifolia; Bamboo; Natural regeneration; Forest species.

\title{
INTRODUÇÃO
}

A Floresta Ombrófila Mista é um ecossistema encontrado normalmente em altitudes elevadas com alto índice pluviométrico durante o ano e contém várias espécies angiospermas e coníferas. Essa formação florestal é conhecida, também, como Floresta de Araucária, pois a Araucaria angustifolia domina o seu dossel superior.

Antes da interferência antrópica e o conseqüente desmatamento das áreas de florestas, a Floresta Ombrófila Mista ocorria nas regiões de clima subtropical, principalmente nos planaltos do Rio Grande do Sul, Santa Catarina e Paraná, e em maciços descontínuos, nas partes mais elevadas de São Paulo, Rio de Janeiro e Sul de Minas Gerais. Entretanto, no Estado do Paraná, resta apenas 1\% da área original de floresta conservada ou em estágio avançado de sucessão (FUPEF-CNPq, 2001).

Para Formento, Schom e Ramos (2004), a exploração da Floresta Ombrófila Mista, quando realizada de forma seletiva, forma grandes clareiras e altera consideravelmente a estrutura da vegetação, sendo que a solução para esse tipo de interferência antrópica é passar pelo processo natural de sucessão secundária. Segundo Guilherme (2000), para que ocorra um melhor desenvolvimento e para atingir a maturidade reprodutiva, a maioria das espécies arbóreas necessita da abertura do dossel, do aumento de incidência luminosa e da disponibilidade de água e nutrientes.

O manejo dessas florestas, para fins produtivos e conservacionistas é uma alternativa viável (SANQUETTA; MATTEI, 2002). Entretanto, para manejar racionalmente essas florestas alteradas por ações antrópicas, é preciso conhecer e respeitar sua capacidade regenerativa. Tal capacidade está intimamente relacionada com três processos demográficos: recrutamento, crescimento e mortalidade os quais governam a sustentabilidade e diversidade da floresta (SANQUETTA, 1996).

Segundo Sanquetta, Dalla Corte e Eisfeld (2003), para manejar racionalmente as florestas 
alteradas por seguidas ações antrópicas, é preciso conhecer e respeitar sua capacidade regenerativa e produtividade. Dessa forma, é necessário o conhecimento dos ecossistemas florestais primários não só para recuperar a vegetação original, mas também para o conhecimento das potencialidades biológicas para o homem das espécies de rápido crescimento (FORMENTO; SCHOM; RAMOS, 2004).

Estudos realizados por Filgueiras (1988), no Chile, Young (1991), no Peru, e Oliveira Filho et al. (1994) e Carvalho (1992), em florestas tropicais do Brasil, corroboram a importância que os bambus exercem como reguladores dos processos de regeneração florestal.

Conforme é mostrado pela literatura, a forte presença competitiva das taquaras no subdossel das florestas de araucária, notadamente naquelas altamente antropizadas, inibe inequivocamente os processos demográficos das populações arbóreas e, conseqüentemente, da comunidade como um todo.

Assim, florestas que sofreram interferência antrópica propiciam o aparecimento de um subbosque bastante denso, onde outras espécies arbóreas e gramíneas se desenvolvem. De acordo com Sanquetta et al. (2005), a presença das taquaras no subdossel das florestas de araucária pode ser o fator causador do decréscimo regenerativo das espécies arbóreas dominantes.

O bambu do gênero Merostachys, mais conhecido como taquara, aparece na América Central e América do Sul, apresentando maior diversidade no Brasil, com aproximadamente 20 espécies (MCCLURE, 1973; FILGUEIRAS, 1988; SODERSTROM et al., 1988; SENDULSKY, 1992; GUILHERME; RESSEL, 2001). De acordo com estudos realizados por Maack (1968), Merostachys e Chusquea, são os gêneros de bambu que mais se destacam na região do Paraná.

Os bambus pertencem à família das gramíneas (Poaceae ou Gramineae), subfamília Bambusoideae, que possui duas subdivisões Bambuseae (lenhosos) e Olyrae (herbáceos). Segundo Londono (1990), os bambus são considerados as gramíneas que possuem a estrutura vegetativa mais complexa e seu tamanho pode variar de 5 centímetros até 30 metros de altura. Para Filgueiras (1988), os bambus se distinguem dos demais membros da família das gramíneas por suas características morfológicas, anatômicas, fisiológicas e ecológicas.

Para Sanquetta et al. (2005), visando à restauração de áreas degradadas no ecossistema da Floresta de Araucária, é indispensável reconhecer o papel que as taquaras exercem sobre a regeneração das espécies arbóreas locais.

Assim, este experimento visa avaliar a regeneração natural na Floresta Ombrófila Mista das espécies arbóreas Piptocarpha angustifolia (vassourão-branco), Vernonia discolor (vassourão-preto), Myrsine umbellata (capororoca), Ilex paraguariensis (erva-mate), Symplocos tenuifolia (maria-mole), entre outras, por meio do tratamento de controle mecânico de taquaras, sendo que as variáveis estudadas foram freqüência e altura média dos indivíduos de cada espécie.

\section{MATERIAIS E MÉTODOS}

O presente experimento visa a avaliar a regeneração natural, o desenvolvimento e dinâmica de espécies arbóreas da Floresta Ombrófila Mista, no Estado do Paraná, Brasil. Esse experimento vem sendo analisado desde novembro de 2002, no município de General Carneiro, no extremo sul do Paraná, próximo da divisa com Santa Catarina, a cerca de $280 \mathrm{~km}$ de Curitiba. As coordenadas geográficas de referência são: $26^{\circ} 20^{\prime} 35^{\prime \prime}$ e $26^{\circ} 26^{\prime} 13^{\prime \prime}$ S, e 51¹9'49” e 5125’29” W. Na Figura 1 está representada a localização da área deste experimento. 


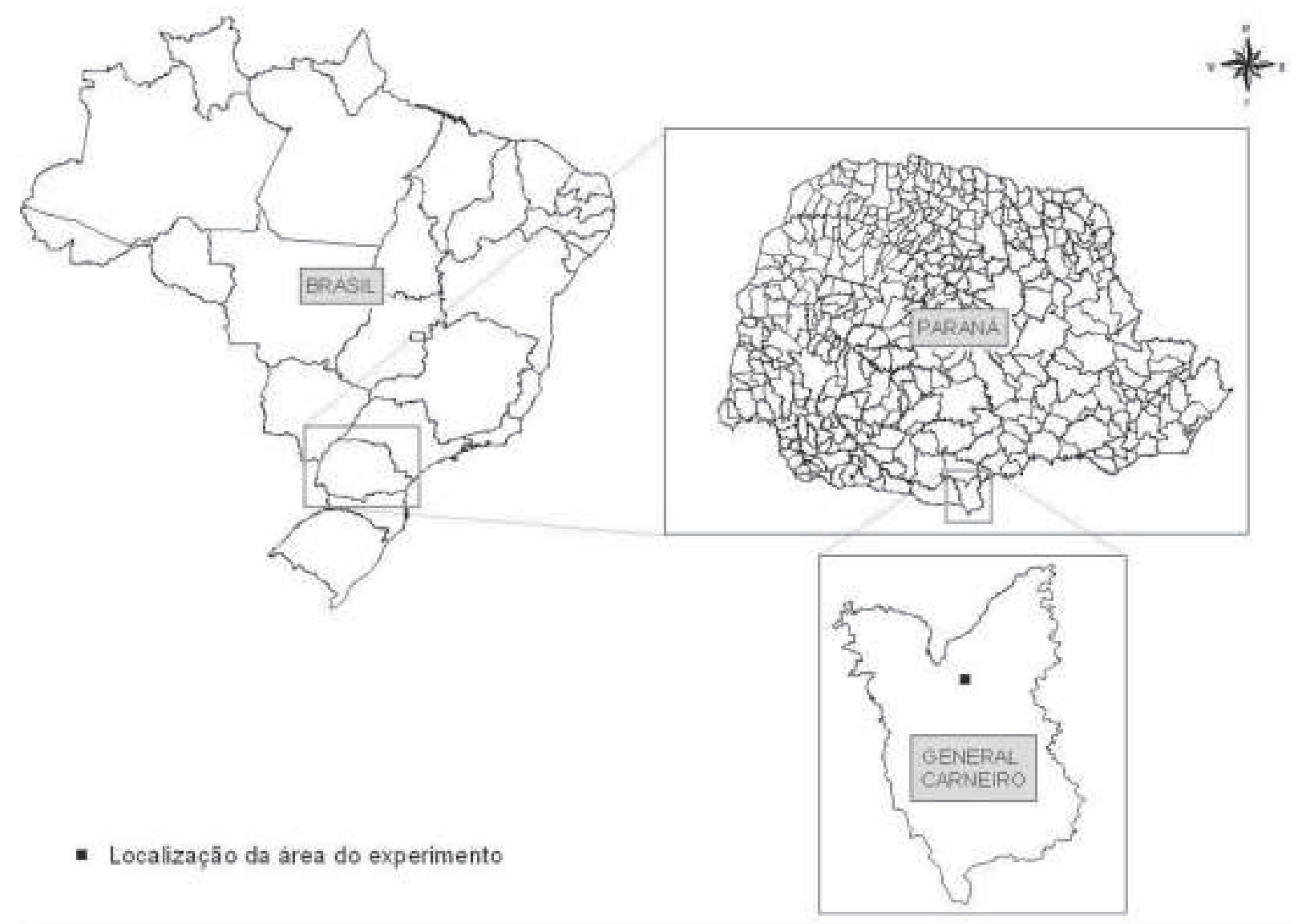

FIGURA 1 - Localização da área do experimento no Brasil

Figure 1 - Experimental area localization in Brazil

O experimento consiste de uma área total de 1 hectare $\left(10.000 \mathrm{~m}^{2}\right)$, subdividida em 25 unidades experimentais de $20 \times 20 \mathrm{~m}$, isto é, com área de $400 \mathrm{~m}^{2}$. Cada unidade experimental recebeu um tratamento de controle mecânico de taquaras, com 5 repetições, sorteadas aleatoriamente, seguindo o delineamento experimental inteiramente casualisado, conforme o esquema na Figura 2. Os tratamentos aplicados neste experimento estão apresentados a seguir:

- T1 - Testemunha;

- $\mathrm{T} 2$ - Corte total da taquara e abandono;

- T3 - Corte total da taquara e retirada;

- T4 - Corte total da taquara e abandono (duas vezes);

- T5 - Corte total da taquara + corte subdossel $(\mathrm{CAP}<31,5 \mathrm{~cm})$, sem retirada. 


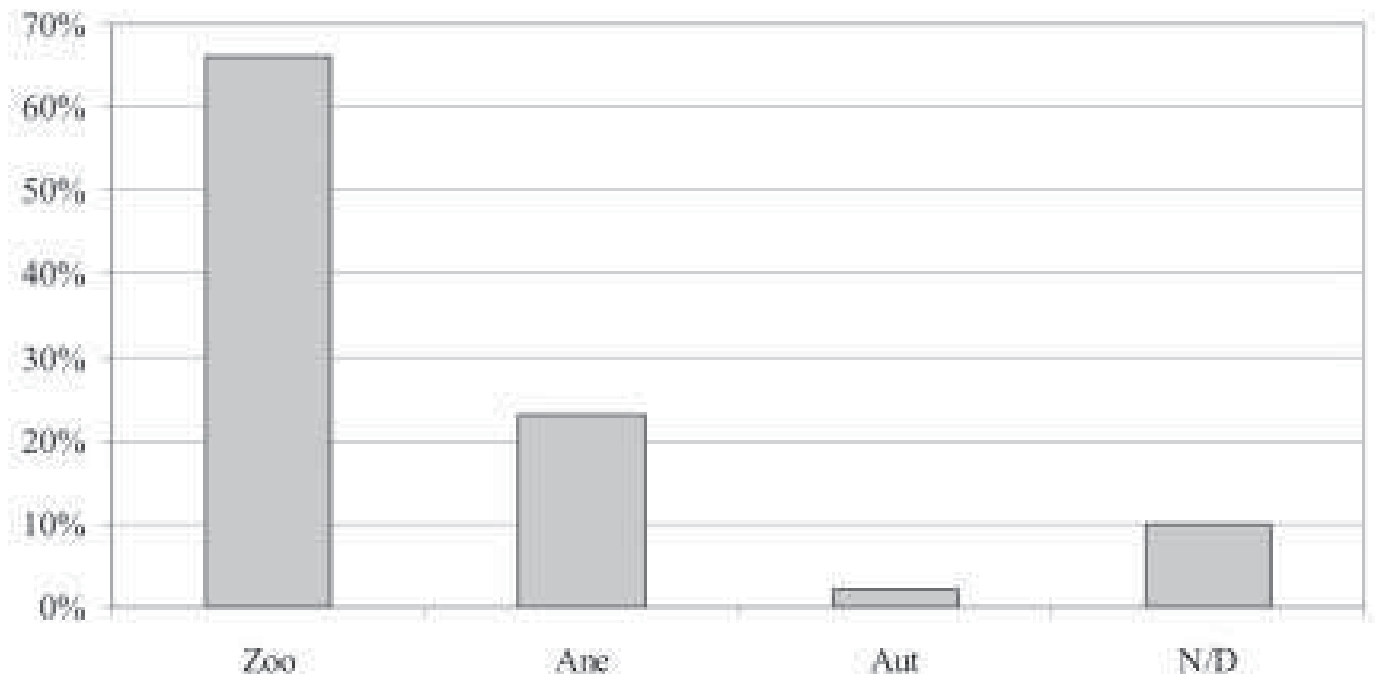

FIGURA 2 - Esquema dos tratamentos aplicados neste experimento Figure 2- Treatments applied in this experiment diagram

Cada unidade experimental foi dividida em duas porções com 20 x $10 \mathrm{~m}\left(200 \mathrm{~m}^{2}\right)$, sendo uma mantida apenas com regeneração natural e a outra adensada com mudas de araucária. O adensamento em parcelas subdivididas consistiu na implementação de 10 fileiras com 5 mudas cada, totalizando 50 mudas em cada subunidade.

As análises foram realizadas avaliando um período de trinta meses a partir do início do experimento, considerando apenas o estabelecimento natural das plântulas de espécies arbóreas na área após a aplicação dos tratamentos. Em uma recente identificação botânica das espécies de taquaras, observou-se que Merostachys multiramea Hackel é a espécie mais abundante e dominante, com mais de 90\% da ocorrência na área. Uma vista fotográfica do experimento está apresentada na Figura 3.

a)

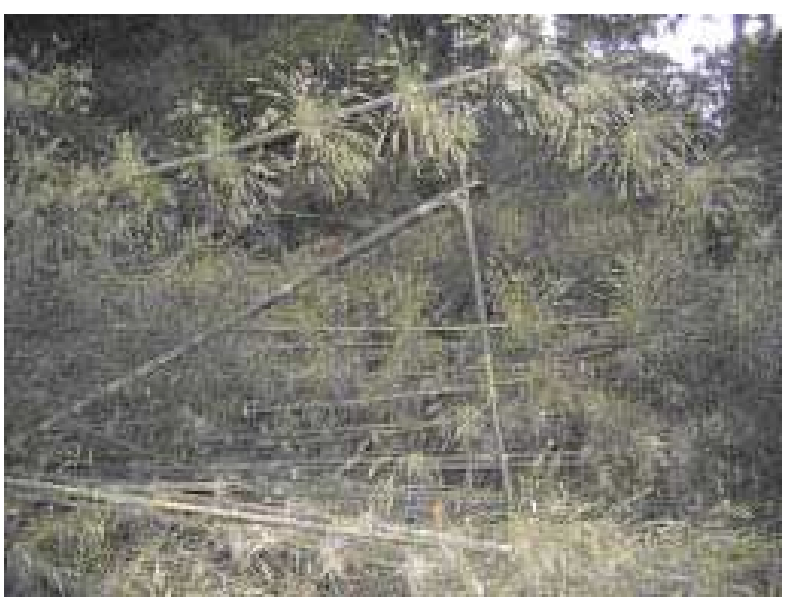

b)

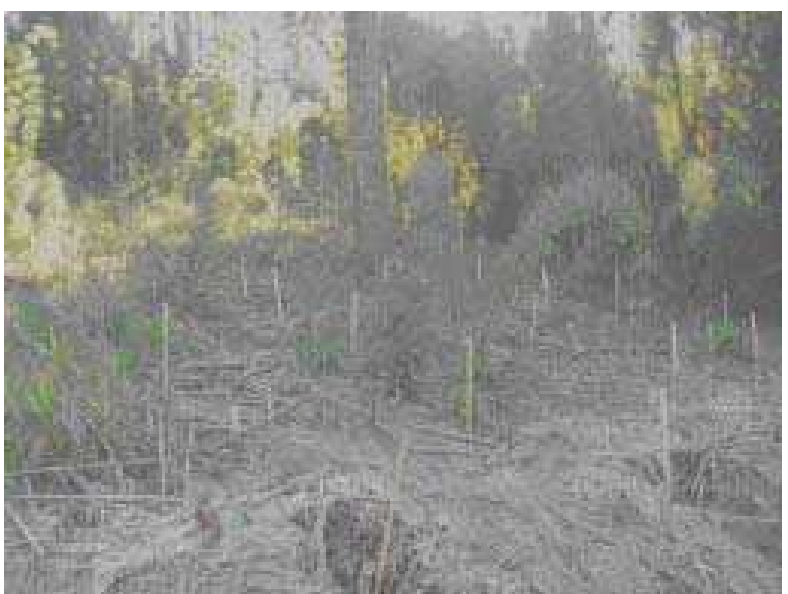

FIGURA 3 - Condições ambientais dos tratamentos com remoção da taquara: (a) testemunha; (b) corte e abandono (primeiro plano); e corte e retirada (segundo plano)

Figure 3 - Environmental condition of the treatments with bamboo removal: (a) control, (b) cut and leaving it in the forest (first layer) and cut with its removal (second layer) 
Em cada uma das repetições, realizou-se a contagem dos indivíduos de regeneração natural, bem como a identificação das espécies, onde cada uma foi marcada com plaquetas de alumínio, com um código específico para cada indivíduo. Foram medidas as alturas com auxílio de paquímetro analógico e trena de mão. A posição de cada indivíduo foi marcada em um croqui para um melhor e efetivo controle nas medições subseqüentes.

As análises realizadas neste experimento visaram a avaliar o efeito dos tratamentos sobre algumas espécies: Piptocarpha angustifolia, Vernonia discolor, Myrsine umbellata, Ilex paraguariensis, Symplocos tenuifolia, a um grupo remanescente, denominado de "outras" e ao total das espécies encontradas no experimento. As variáveis avaliadas foram: freqüência de indivíduos de cada espécie e suas alturas médias. Para a constatação dos efeitos dos tratamentos, foi utilizada análise estatística paramétrica, utilizando-se do Software Excel, da empresa Microsoft.

O primeiro passo foi testar a homogeneidade de variância para cada conjunto de dados analisados, aplicando o teste de Bartlett (1). A espécie Vernonia discolor não apresentou homogeneidade de variância para a variável número de indivíduos, optando-se, neste caso, por realizar apenas uma avaliação gráfica sobre sua dispersão. Testou-se ainda a homogeneidade de variância entre as repetições para a variável altura das regenerações de cada espécie. Nesse caso, a Myrsine umbellata foi a única espécie que apresentou homogeneidade de variância, optando-se, em conseqüência, por uma avaliação apenas gráfica dessa variável para os demais grupos de espécies.

Após a aplicação do teste de Bartlett (1), realizou-se a análise de variância (ANOVA) para a comparação de médias dos tratamentos de cada espécie. A espécie Vernonia discolor foi analisada apenas graficamente por não ter resultado em homogeneidade de suas variâncias.

$$
\chi^{2}=2,3026 x\left[\log \left(\bar{s}^{2}\right) x\left(\sum G L_{i}\right)\right]-\sum\left[\left(G L_{i}\right) x\left(\log s_{i}{ }^{2}\right)\right]
$$

Onde:

$$
\begin{aligned}
& \text { 2,3026 = Constante; } \\
& \chi^{2}=\text { Qui Quadrado; } \\
& \left(\bar{s}^{2}\right)=\text { Média das variâncias; } \\
& G L_{i}=\text { Graus de liberdade; } \\
& s_{i}{ }^{2}=\text { Variância de cada tratamento. }
\end{aligned}
$$

Posteriormente, realizou-se uma avaliação das alturas médias dos indivíduos das principais espécies presentes em cada tratamento, sendo efetuada a ANOVA apenas para a espécie Myrsine umbellata e uma avaliação gráfica para as demais espécies, dado ter sido inviável homogeneizar suas variâncias.

Em conseqüência dos resultados da ANOVA, para as variáveis número de indivíduos e sua altura média, aplicou-se o teste de Tukey.

\section{RESULTADOS E DISCUSSÃO}

\section{Número de indivíduos}

O primeiro resultado gerado neste estudo foi o número de indivíduos e a altura média dos indivíduos encontrados em cada tratamento (TABELA 1): 
TABELA 1 - Número de indivíduos e suas alturas médias em cada tratamento Table 1 - Number of individuals and its average heights in each treatment

\begin{tabular}{|c|c|c|c|c|c|c|c|}
\hline \multirow{2}{*}{ Espécies } & \multirow{2}{*}{ Variável } & \multicolumn{5}{|c|}{ Tratamentos } & \multirow[b]{2}{*}{ Total } \\
\hline & & $\mathrm{T} 1$ & $\mathrm{~T} 2$ & T3 & T4 & T5 & \\
\hline \multirow{2}{*}{ Myrsine umbellata } & $\mathrm{N}^{\circ}$ de indivíduos & 20 & 77 & 46 & 93 & 100 & \multirow[t]{2}{*}{336} \\
\hline & $\mathrm{H}$ média $(\mathrm{cm})$ & 81,4 & 91,4 & 97,2 & 107,8 & 96,5 & \\
\hline \multirow{2}{*}{ Ilex paraguariensis } & $\mathrm{N}^{\circ}$ de indivíduos & 164 & 136 & 113 & 227 & 212 & \multirow[t]{2}{*}{852} \\
\hline & $\mathrm{H}$ média $(\mathrm{cm})$ & 104,1 & 100,5 & 119,9 & 122,6 & 108,4 & \\
\hline \multirow{2}{*}{ Symplocos tenuifolia } & $\mathrm{N}^{\circ}$ de individuos & 36 & 26 & 27 & 43 & 53 & \multirow[t]{2}{*}{185} \\
\hline & $\mathrm{H}$ média $(\mathrm{cm})$ & 151,1 & 133,3 & 148,3 & 151,4 & 132,9 & \\
\hline \multirow{2}{*}{ Piptocarpha angustifolia } & $\mathrm{N}^{\circ}$ de indivíduos & 31 & 175 & 657 & 134 & 113 & \multirow[t]{2}{*}{1110} \\
\hline & $\mathrm{H}$ média $(\mathrm{cm})$ & 111,5 & 184,2 & 202,3 & 176,4 & 159,7 & \\
\hline \multirow{2}{*}{ Vernonia discolor } & $\mathrm{N}^{\circ}$ de indivíduos & 42 & 224 & 321 & 101 & 248 & \multirow[t]{2}{*}{936} \\
\hline & $\mathrm{H}$ média $(\mathrm{cm})$ & 154,2 & 210,0 & 195,4 & 170,4 & 181,6 & \\
\hline \multirow{2}{*}{ Outras } & $\mathrm{N}^{\circ}$ de individuos & 150 & 123 & 151 & 292 & 139 & \multirow[t]{2}{*}{855} \\
\hline & $\mathrm{H}$ média $(\mathrm{cm})$ & 88,1 & 84,6 & 90,6 & 84,6 & 78,7 & \\
\hline
\end{tabular}

Pelo teste de Bartlett, realizou-se a análise de homogeneidade de variância para os dados em questão. Na Tabela 2, observa-se o resultado desse teste para cada uma das espécies avaliadas com relação à variável número de indivíduos. De acordo com esse teste, as variâncias do número de indivíduos de todas as espécies foram consideradas homogêneas, exceto para a espécie Vernonia discolor. Para essa espécie, optou-se por realizar apenas uma apresentação gráfica do número de indivíduos por tratamento.

TABELA 2 - Teste de Bartlett aplicado ao número de indivíduos

Table 2 - Bartlett test applied to number of individuals

\begin{tabular}{lcc}
\hline Nome vernacular & Bartlett & $\mathrm{p}$ \\
\hline Myrsine umbellata & 1,22585 & 0,447766 \\
Ilex paraguariensis & 1,6497 & 0,058607 \\
Symplocos tenuifolia & 1,02459 & 0,983065 \\
Piptocarpha angustifolia & 1,12316 & 0,736875 \\
Outras & 1,15917 & 0,611748 \\
\hline
\end{tabular}


Observa-se na Tabela 3 que as únicas espécies que apresentaram diferenças significativas entre as médias, de acordo com a ANOVA, em relação aos tratamentos aplicados, foram: Myrsine umbellata e Piptocarpha angustifolia.

TABELA 3 - Análise de variância aplicada ao número de indivíduos

Table 3 - Analysis of variance applied to number of individuals

\begin{tabular}{lcc}
\hline Nome vernacular & $\mathrm{F}$ & $\mathrm{p}$ \\
\hline Myrsine umbellata & 4,43 & $0,0100^{*}$ \\
Ilex paraguariensis & 0,99 & 0,4360 \\
Symplocos tenuifolia & 0,13 & 0,9703 \\
Piptocarpha angustifolia & 5,80 & $0,0032^{*}$ \\
Outras & 0,98 & 0,4408 \\
\hline
\end{tabular}

*Diferença significativa a 95\% de probabilidade.

Portanto, havendo diferenças significativas em relação aos tratamentos para algumas espécies, buscou-se identificá-las pelo teste de médias. O teste de média adotado foi o de Tukey e os resultados encontrados para a espécie Myrsine umbellata estão apresentados na Tabela 4 e para a espécie Piptocarpha angustifolia na Tabela 5. No caso da espécie Myrsine umbellata, percebeu-se que os tratamentos 1 e 3 (testemunha e corte total e retirada da taquara) apresentaram comportamentos semelhantes, sendo agrupados em um primeiro grupo 1 . Os outros tratamentos 4, 5, 2 e 3 apresentaram comportamentos semelhantes e foram agrupados em um segundo grupo. Para a espécie Piptocarpha angustifólia, pode-se afirmar que os tratamentos 1, 2, 4 e 5 tiveram comportamentos semelhantes, formando um grupo 1, e os tratamentos 2, 3 e 4 tiveram outro comportamento, porém semelhante entre eles, formando um grupo 2. Os resultados permitem concluir que os tratamentos 2 e 4 são semelhantes tanto para o grupo 1 quanto para o grupo 2.

TABELA 4 - Teste de Tukey aplicado ao número de indivíduos da espécie Myrsine umbellata Table 4 - Tukey test applied to the number of individuals of Myrsine umbellata

\begin{tabular}{ccc}
\hline Tratamentos & Grupos \\
& 1 & 2 \\
\hline 1 & $\mathrm{~A}$ & $\mathrm{~B}$ \\
3 & $\mathrm{~A}$ & $\mathrm{~B}$ \\
2 & & $\mathrm{~B}$ \\
5 & & $\mathrm{~B}$ \\
4 & & \\
\hline
\end{tabular}


Foram realizadas análises para a espécie Vernonia discolor, porém, dada a significância observada para número de indivíduos por parcela, tentou-se homogeneizar as variâncias aplicandose as transformações dos dados, conforme sugerido pela literatura, porém não se conseguiu sucesso em nenhum dos casos, razão pela qual optou-se por realizar apenas uma análise gráfica para essa espécie (GRÁFICO 1).

TABELA 5 - Teste de Tukey aplicado ao número de indivíduos da espécie Piptocarpha angustifolia Table 5 - Tukey test applied to the number of individuals of Piptocarpha angustifolia

\begin{tabular}{ccc}
\hline Tratamentos & \multicolumn{2}{c}{ Grupos } \\
& 1 & 2 \\
\hline 1 & A & \\
2 & A & \\
4 & A & B \\
3 & A & B \\
& & B \\
\hline
\end{tabular}

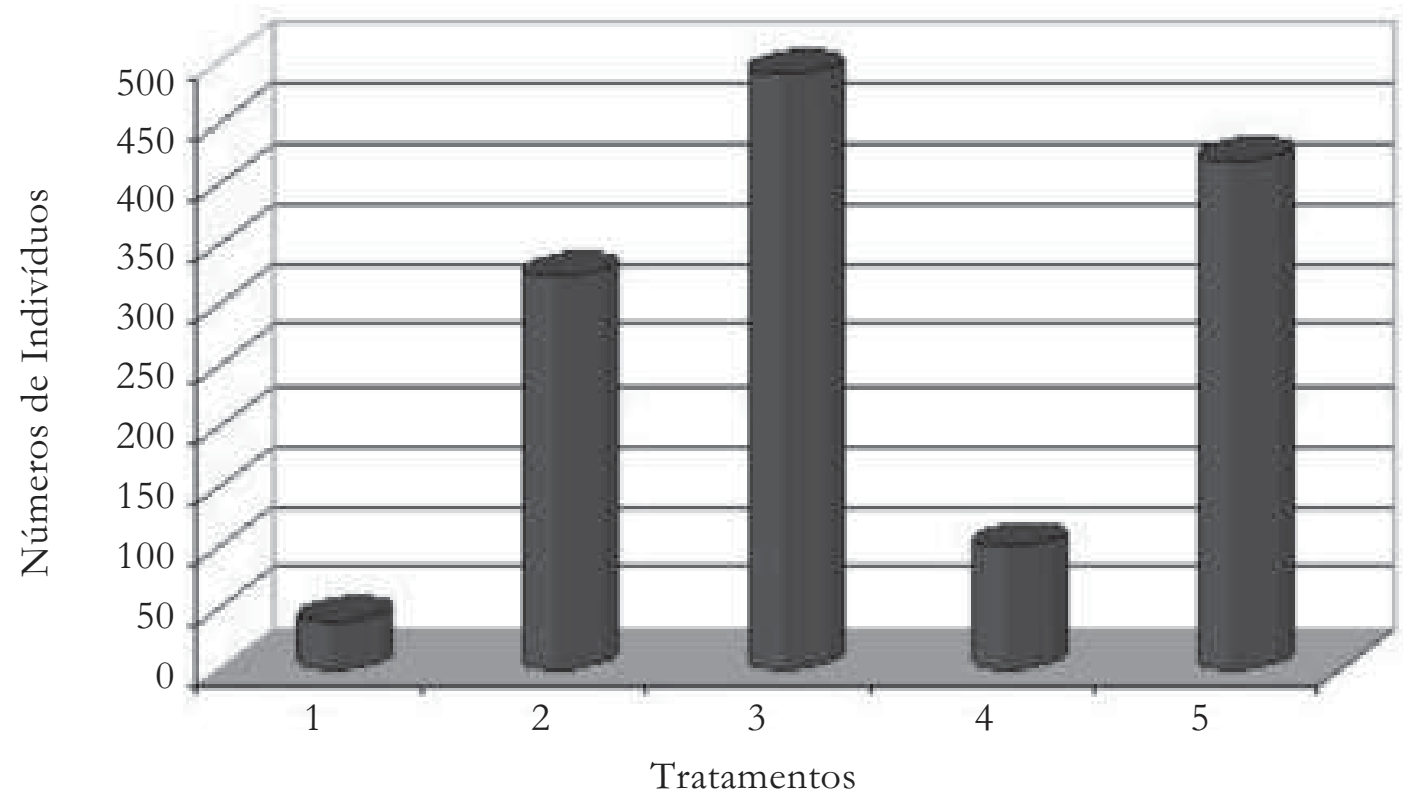

GRÁFICO 1 - Número médio de indivíduos por tratamento para a espécie Vernonia discolor Graph 1 - Average number of individuals per treatment of Vernonia discolor 
Apesar de não se avaliar estatisticamente, os resultados numéricos observados graficamente revelam que existem diferenças em relação ao número de indivíduos nos diferentes tratamentos. Verificou-se que no tratamento 3 (corte total da taquara e retirada) ocorreu o maior número de indivíduos (321), seguido do tratamento 5 (corte total da taquara + corte subdossel, sem retirada) (248).

\section{Altura média dos indivíduos}

A única espécie que apresentou homogeneidade de variância para a altura média foi a Myrsine umbellata (TABELAS 6 e 7). Observou-se que nessa espécie não houve diferenças significativas para a variável altura média em relação aos tratamentos aplicados no experimento. As variâncias das demais espécies não foram homogêneas, de acordo com o teste de Bartlett, mesmo após a transformação dos dados. Dessa forma, optou-se por utilizar apenas uma análise gráfica das alturas médias por tratamentos.

TABELA 6 - Teste de Bartlett aplicado à espécie Myrsine umbellata Table 6 - Bartlett test applied to Myrsine umbellata

\begin{tabular}{lcc}
\hline Nome vernacular & Bartlett & $\mathrm{p}$ \\
\hline Myrsine umbellata & 1,243 & 0,412133 \\
\hline
\end{tabular}

TABELA 7 - Análise de variância para a espécie Myrsine umbellata

Table 7 - Analysis of variance to Myrsine umbellata

\begin{tabular}{lcc}
\hline Nome vernacular & $\mathrm{F}$ & $\mathrm{p}$ \\
\hline Myrsine umbellata & 1,88 & 0,1537 \\
\hline
\end{tabular}

No Gráfico 2 estão plotadas as alturas médias para cada espécie em cada um dos tratamentos. Nesse gráfico, observou-se que as espécies Piptocarpha angustifolia e Vernonia discolor obtiveram as maiores alturas médias em quase todos os tratamentos. Isso possivelmente se deve ao hábito dessas espécies de colonizarem rapidamente os ambientes. Por outro lado, a espécie Myrsine umbellata e o grupo "Outras" foram as que apresentaram as menores alturas médias. 


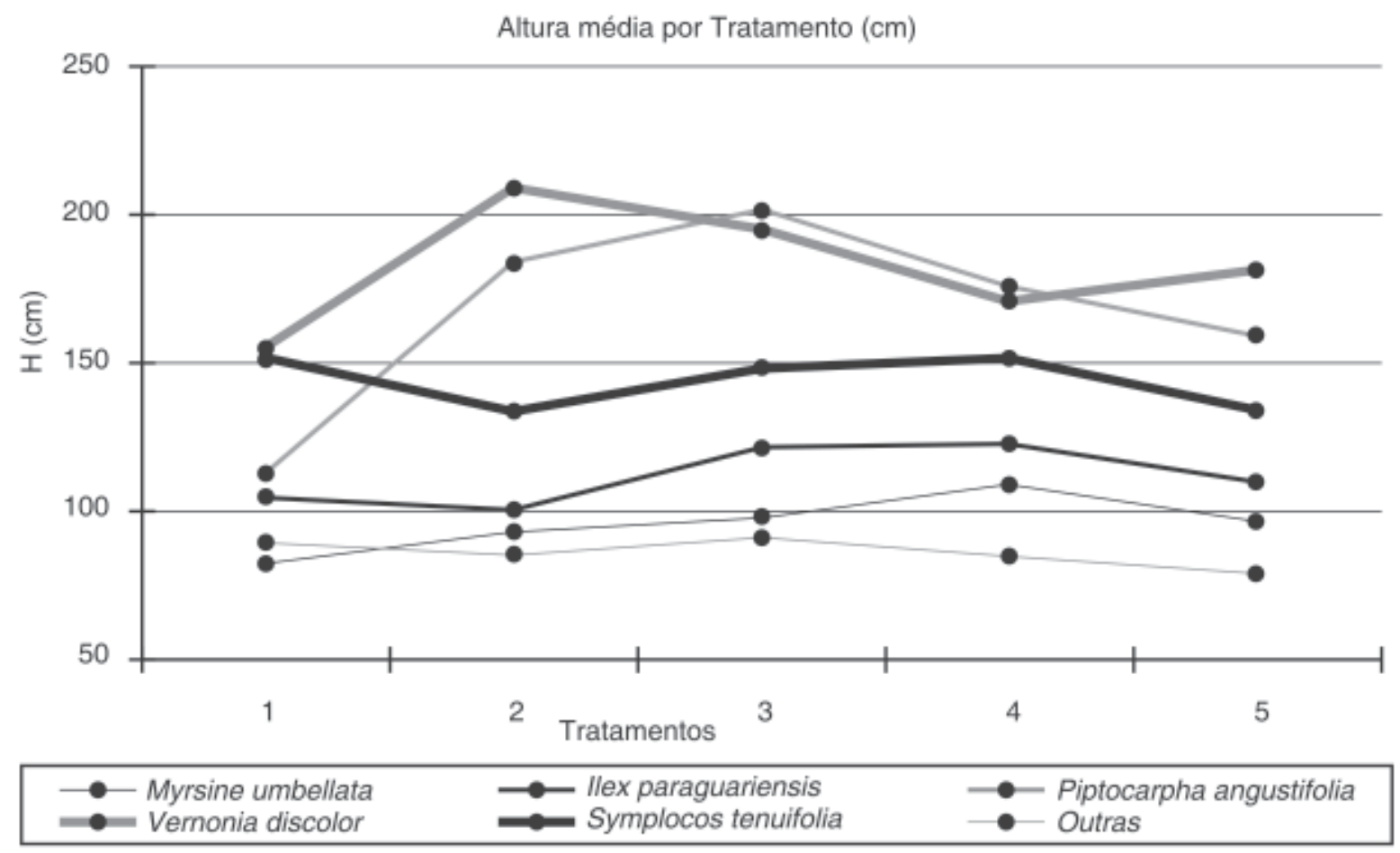

GRÁFICO 2 - Alturas médias das espécies nos tratamentos realizados Graph 2 - Average heights of the species in the applied treatments

\section{CONCLUSÕES}

$\mathrm{Na}$ presente pesquisa, as únicas espécies que resultaram em diferenças significativas para número de indivíduos por parcela em presença de taquara foram Myrsine umbellata e Piptocarpha angustifolia. Para a Myrsine umbellata, os tratamentos que diferiram foram: testemunha e corte total com retirada da taquara.

Com relação às alturas médias das espécies, não foi possível realizar análises estatísticas paramétricas devido à falta de homogeneidade de variâncias, exceto para a espécie Myrsine umbellata, entretanto os resultados da análise de variância aplicada aos seus dados mostraram não significância para as médias das alturas por tratamento. Portanto, ao se avaliar graficamente os valores médios das alturas nos tratamentos, observou-se que as espécies Piptocarpha angustifolia e Vernonia discolor atingiram as maiores alturas médias em todos os tratamentos aplicados, fato este explicado pelo hábito colonizador de ambientes dessas espécies.

As taquaras da espécie Merostachys multiramea auxiliam a preservar as funções ecológicas da floresta, pois os resultados do experimento demonstram que, quanto mais as taquaras dessa espécie são suprimidas, mais evidentes se tornam os processos de sucessão ecológica, tendendo a encontrar um equilíbrio na floresta. A partir disso, é necessário se descobrir quais os tratamentos de controle mecânico de taquaras são mais eficientes para acelerar esse processo de sucessão ecológica, garantindo, assim, melhores usos dos recursos naturais, sejam eles visando a objetivos comerciais ou preservacionistas.

Para maior consistência dos conhecimentos dos processos dinâmicos envolvendo as espécies de taquaras, será relevante a continuidade do monitoramento do experimento, bem como proceder avaliações sazonais durante o ano. Conseqüentemente, é importante mencionar que os resultados apresentados neste trabalho são apenas parciais. 


\section{REFERÊNCIAS}

CARVALHO, J. O. P. Structure and dynamics of a logged-over brazilian amazonian rain forest. Oxford: Oxford University, 1992.

FILGUEIRAS, T. S. A floração dos bambus e seu impacto ecológico. Eugeniana, Nova Friburgo, v. 15, p. 1-8, 1988.

FORMENTO, S.; SCHOM L. A.; RAMOS, R. A. B. Dinâmica estrutural arbórea de uma Floresta Ombrófila Mista em Campo Belo do Sul, SC. Cerne, Lavras, v. 10, n. 2, p. 196-212, jul./dez. 2004.

FUPEF-CNPq (Fundação de Pesquisas Florestais do Paraná - Conselho Nacional de Desenvolvimento Científico e Tecnológico). Conservação do bioma floresta com araucária. Curitiba: Relatório Final, v. $1 / 2,2001.456$ p.

GUILHERME, F. A. G. Efeitos da cobertura de dossel na densidade e estatura de gramíneas e da regeneração natural de plantas lenhosas em mata de galeria, Brasília -DF. Cerne, Lavras, v. 6, n. 1, p. 60-66, 2000.

GUILHERME, F. A. G.; RESSEL, K. Floral biology and breeding system of Merostachys riedeliana (Poaceae: Bambusoideae). Revista Brasileira de Botânica, São Paulo, v. 24, n. 2, 2001. Disponível em:

<http://www.scielo.br/scielo>. Acesso em: 20 jul. 2006.

LONDONO, X. Aspectos sobre la distribucion y la ecologia de los bambues de Colombia (Poaceae: Bambusoideae). Caldasia, v. 16, n. 77, p. 139-153, 1990.

MAACK, R. Geografia física do estado do Paraná. Curitiba: CODEPAR, 1968. 350 p.

MCCLURE, F. A. Genera of bamboos native to the new world (Gramineae: Bambusoideae). Smithsonian Contributions to Botany. v. 9, p. 1-148, 1973.

OLIVEIRA FILHO, A. T. et al. Effect of flooding regime and understorey bamboos on the physiognomy and tree species composition of a tropical semideciduous forest in southeastern Brazil. Vegetatio, The Hague, v. 113, p. 99-124, 1994.

SANQUETTA, C. R. et al. Crescimento, mortalidade e recrutamento em duas Florestas de Araucária (Araucária angustifolia (Bert.) O. Ktze.) no Estado do Paraná, Brasil. Revista Ciências Exatas e Naturais, v. 5, n. 1, p. 101-112, 2003.

SANQUETTA, C. R. et al. Estabelecimento de plântulas de espécies arbóreas em um experimento de controle de taquaras (Bambusoideae) no sul do Paraná, Brasil. Curitiba: UFPR, 2005.

SANQUETTA, C. R.; MATTEI, E. Manejo racional da floresta de araucária. Meio Ambiente Santa Catarina, Florianópolis, v. 2, p. 58, 2002.

SANQUETTA, C. R. Fundamentos biométricos dos modelos de simulação florestal. Curitiba: FUPEF, 1996. 49 p. (Série Didática, n. 8).

SENDULSKY, T. Merostachys burmanii (Poaceae: Bambusoideae: Bambuseae), a new species from Brazil. Novon, v. 2, p. 111-113. 1992.

SODERSTROM, T. R.; JUDZIEWICZ, E. J.; CLARK, L. G. Distribution patterns of neotropical bamboos. In: Proceedings of a workshop on neotropical distribution patterns. Academia Brasileira de Ciências, Rio de Janeiro: Edição dos autores, 1988. p. 121-157.

YOUNG, K. R. Natural history of an understory bamboo (Chusquea sp.) a tropical timberline forest. Biotropica, St. Louis, v. 23, n. 4b, p. 542-554, 1991. 